\title{
Appetite for sugar and fat in the thiamine deficient rat'
}

H. R. BEHRMAN AND OWEN MALLER

NORTH CAROLINA STATE UNIVERSITY AT RALEIGH

\begin{abstract}
Abstraet
Thiamine deficient and control rats were permitted access to either sucrose solution or an isocaloric corn-oil emulsion for a 32 day period. The deficient animals increased, rather than reduced, their intake of the sucrose solution and 5 out of 7 animals failed to show an increase in consumption of the corn-oil emulsion.
\end{abstract}

\section{Problem}

Richter \& Hawkes (1941) and Scott et al (1949) observed that the thiamine deficient rat would, in a multiple choice situation, increase fat and decrease sugar intake. Such an increase in fat and decrease in carbohydrate intake would be advantageous to the thiamine deficient animal since it is believed that the thiamine requirement for the metabolism of carbohydrate is higher than it is for fat (Goldsmith, 1964). However, the above studies are difficult to interpret because the reported change in appetite occurred either as a consequence of a general B-vitamin deficiency or in a multiple-choice situation in which an aversion to one of the food components might produce a spurious preference for another. The present study attempted to take these factors into account by studying only thiamine deficiency and by employing a two-choice situation. The question asked was would the rat decrease sugar and increase fat intake to alleviate a thiamine deficiency?

\section{Method}

Thirty male rats of the Holtzman strain ranging in weight between 175-200 gm, were randomly assigned to one of four groups; two experimental groups $(\mathrm{N}=7)$ and two control groups $(\mathrm{N}=8)$. The animals were individually caged and maintained in a temperature and light controlled room. Water and food were available ad libitum. All animals were adjusted to a semisynthetic adequate diet for 16 days prior to the beginning of the experiment. During the last 5 days of the adjustment period and for the duration of the experiment the animals were given a choice between a $15 \%$ sucrose solution and distilled water, or an isocaloric corn oil-tween 80 emulsion (approximately $7 \%$ ) and distilled water. One experimental and control group received corn oil. These are referred to as the thiamine deficient-fat group (TDF) and the thiamine replete-fat group (TRF). The groups receiving the sucrose solution are referred to as TDS and TRS groups, respectively。

Following the adjustment period to the diet and to the solutions, TDF and TDS groups were placed on a thiamine deficient diet which was identical to the diet for TRF and TRS groups but lacked thiamine hydrochloride. This regimen was continued for a 32 day period at the end of which, purified thiamine hydrochloride was added to the deficient diet to demonstrate that the TDF and TDS groups were deficient in thiamine.

Intake measurements were recorded daily, and body weight measurements were recorded every third day. The corn oil emulsion and sucrose solutions were prepared on a weight/volume basis, e.g., $15 \mathrm{gm}$ of sucrose made up to a total volume of $100 \mathrm{ml}$ with distilled water. The sucrose solutions and the corn oil emulsion were changed every second day.

\section{Results}

The symptoms of thiamine deficiency, i.e., anorexia and concomitant growth retardation, appeared within the first two weeks in animals on the deficient diet. The lower portion of Figs. 1 and 2 depict the corn oil and sucrose intake of the thiamine deficient and adequate animals. The intake of corn oil emulsion and the sucrose solution is expressed as a percentage of the total daily caloric intake. The upper portion of the figures display

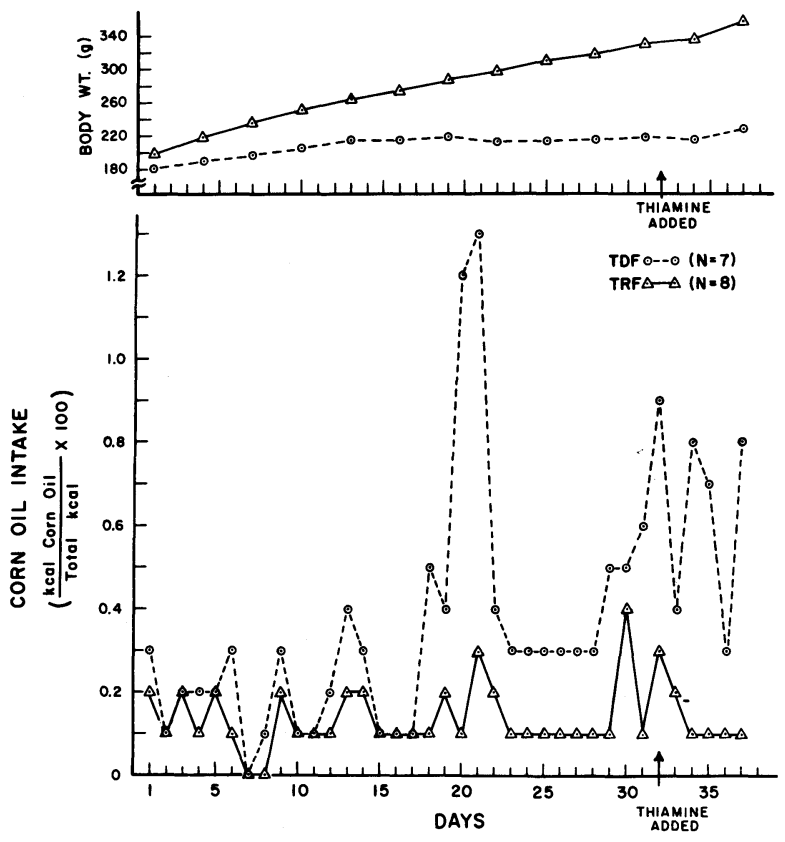

Fig. 1. Corn oil intake, expressed as a percentage of total caloric intake, and growth rate for thiamine deficient and replete rats. 

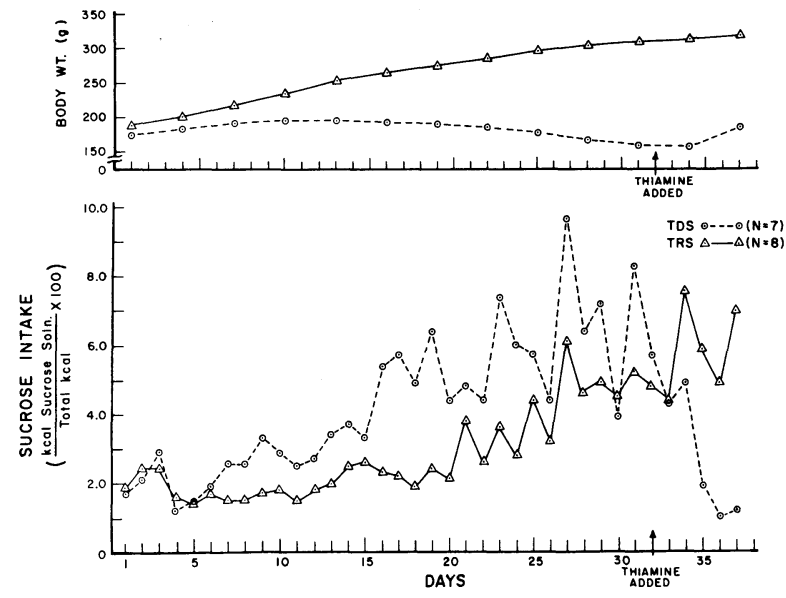

Fig. 2. Sucrose intake expressed as a percentage of total caloric intake, and growth rate for thiamine deficient and replete rats. the growth of the respective groups during the course of the experiment.

The TDF group showed a significant increase, during the last 10 days of the experimental period as compared to the first 10 days, in the percent of calories ingested from the corn oil emulsion $(p<.01)$. However, the overall increase in fat consumption by the TDF group is the result of an increased intake in only 2 of the 7 animals. The corn oil intake of the TRF group remained relatively stable at $.1 \%$ of their total caloric intake. At the end of the deficiency period (32 days) the TDF group had a mean body weight of $214 \mathrm{gm}$ as compared to $334 \mathrm{gm}$ for the TRF group.

In the case of the TDS animals, there was a significant increase in the calories ingested from the sucrose, comparing the first 10 days to the last 10 days of the experimental period $(\mathrm{p}<.01)$. The difference in sucrose intake between the TDS and TRS was also significant $(p<.01)$. The total caloric intake of the deficient group however, was less than that of the controls, because these animals consumed less of the dry diet. At the end of the deficiency period the TDS group had a mean body weight of $157 \mathrm{gm}$ as compared to $314 \mathrm{gm}$ for the TRS group. Three of the seven animals in the TDS died after the 33rd day of deficiency, no other deaths occurred in any of the other groups.

Both the TDF and TRF groups showed a greater growth rate and intake of dry diet than did their respective counterparts, the TDS and TRS groups. Although the sucrose solution and corn oil emulsion were isocaloric a much greater proportion of calories from the dry diet was ingested by the TRF and TDF groups. The TDS and TRS animals, due to a high intake of calories from sucrose, limited their intake of other essential nutrients provided by the dry diet. This reduction was considerably greater for the deficient animals.

\section{Diseussion}

The increase in fat intake of the deficient animals represented only a small proportion of the total caloric intake and was notobserved in five of the seven animals. It is possible that the corn-oil emulsion was not palatable and as a result the animals chose to meet their caloric requirements by ingesting more of the dry diet. Thus, the data fail to support the increased fat intake observed by Richter \& Hawkes (1941) and Scott et al (1950).

However, sucrose was equally acceptable to both the TDS and TRS groups. The data described indicate that the thiamine deficient rat would not restrict its intake of sucrose. In fact, the percentage of calories ingested from the sucrose solutions increased rather than decreased in the TDS group. This finding is not in agreement with Richter \& Hawkes (1941), but these investigations used solid foodstuffs and employed a multiple vitamin deficiency, which may account for the different results obtained. Furthermore, it is possible that the thiamine deficiency caused an increased aversion to the diet. Thus, the deficient animals continued to ingest the sucrose even though it was detrimental to their growth and aggravated their thiamine deficiency.

It is possible that the response to sugar, established before the deficiency became severe, potentiated the low caloric intake of dry diet relative to the sucrose solution by the TDS animals. Dietary habits have been shown to interefere with the correction of deficiencies. For example, Harriman (1955) found that rats with sugar available prior to adrenalectomy failed to shift their preference from sugar to a saline solution and consequently died. In the present experiment the control animals with access to corn oil showed a higher growth rate than did the control animals which received sucrose, further suggesting the potency of palatability and/or dietary habits in the correction of a nutritional deficiency.

\section{References}

Goldsmith, G. A.The B vitamins: Thiamine, Riboflavin, Niacin. In: Beaton, G. H., \& McHenry, E. W. (Eds.), Nutrition. Vol. II, New York: Academic Press, 1964.

Harriman, A. The effect of a preoperative preference for sugar over salt upon compensatory salt selection by adrenalectomized rats. J. Nutr. 1955, 57, 271-276.

Richter, C. P., \& Hawkes, C. D. The dependence of carbohydrate, fat and protein appetite on various components of the vitamin B complex. Amer. J. Physiol., 1941, 131, 639-649.

Scott, E. M., Verney, E. L., \& Morrissey, P. D. Self selection of the diet. XII. Effects of B vitamin deficiencies on selection of food components. J. Nutr., 1950, 41, 373-381.

Notes

1. This work was supported, in part, by National Institutes of Health, grant No. NB-03896 and USPHS fellowship 2F2 HD-21-201-2 from National Institute of Child Health and Human Development.

2. The vitamins were kindly donated by Hoffman-LaRoche, Inc. 